\title{
A smoothness criterion for monotonicity-preserving subdivision
}

\author{
Michael S. Floater*, Carolina Beccari \\ Tom Cashman $\stackrel{\ddagger}{\ddagger}$ and Lucia Romani ${ }^{\S}$
}

August 1, 2012

\begin{abstract}
In this paper we study subdivision schemes that both interpolate and preserve the monotonicity of the input data, and we derive a simple ratio condition that guarantees the continuous differentiability of the limit function. We then show that the condition holds for both a scheme of Kuijt and van Damme, based on rational functions, and a scheme of Sabin and Dodgson, based on square roots.
\end{abstract}

$M S C: 65 D 05,65 D 17$

Keywords: Interpolatory subdivision, monotonicity-preservation

\section{Introduction}

Given a sample of function values $f_{k}=f(k), k=0,1,2, \ldots, n$, of a function $f:[0, n] \rightarrow \mathbb{R}$, one way to construct an interpolant, i.e., a function

${ }^{*}$ Centre of Mathematics for Applications, Department of Informatics, University of Oslo, PO Box 1053, Blindern, 0316 Oslo, Norway, email: michaelf@ifi.uio.no

${ }^{\dagger}$ Dipartimento di Matematica, Universita di Bologna, P.zza di Porta S.Donato 5, 40127 Bologna, Italy, email: beccari@dm.unibo.it

${ }^{\ddagger}$ Faculty of Informatics, University of Lugano, Via Giuseppe Buffi 13, 6904 Lugano, Switzerland, email: thomas.cashman@usi.ch

${ }^{\S}$ University of Milano-Bicocca, Dept. of Mathematics and Applications, Via R. Cozzi, 53, 20125 Milano, Italy, email: lucia.romani@unimib.it 
$g:[0, n] \rightarrow \mathbb{R}$ such that $g(k)=f_{k}$, is to use subdivision. In this paper we study subdivision schemes that preserve monotonicity in the sense that they generate an interpolant that is increasing, i.e., $g(x)<g(y)$ for $x<y$, whenever $f$ is increasing.

Monotonicity is a natural shape property to preserve, and various methods address this problem using piecewise polynomials and rational functions $[5,11,12,13]$. The use of subdivision to achieve this $[15,16]$ has recently gained greater focus because monotonicity is required for the parameter values used when interpolating points with a parametric curve. If a subdivision method is used to construct the curve, such as in $[1,2,7,17]$, the parameter values will themselves satisfy a subdivision scheme which should preserve monotonicity. As suggested in [17], it might be desirable that these parameter values become 'smoothed out' in some sense in the limit, which we can interpret as saying that the scheme for the parameter values should have at least $C^{1}$ smoothness.

In this paper we derive a simple ratio condition that guarantees the continuous differentiability of the limit function. By applying this condition, we show that both a scheme proposed by Kuijt and van Damme and a scheme proposed by Sabin and Dodgson are $C^{1}$, and could therefore be applied to smooth parameter values used for curve subdivision.

\section{A ratio condition}

Both of the schemes we analyze are four-point schemes, and so in both cases we start by adding two data at each end, so that we have increasing data $f_{k}$ for $-2 \leq k \leq n+2$, the extra data acting as 'boundary conditions'. We now initialize the scheme by setting $g_{0, k}=f_{k}, k=-2, \ldots, n+2$, and then for each $j \in \mathbb{N}_{0}$ we generate data by the rules

$$
g_{j+1,2 k}=g_{j, k}, \quad g_{j, k}<g_{j+1,2 k+1}<g_{j, k+1} .
$$

For each $j$ let $g_{j}$ be the polygon through the data $\left(2^{-j} k, g_{j, k}\right)$. The limit function, if it exists, is then

$$
g(x):=\lim _{j \rightarrow \infty} g_{j}(x), \quad x \in[0, n] .
$$

Our approach to the convergence analysis is to study the behaviour of the differences

$$
d_{j, k}=g_{j, k+1}-g_{j, k}>0
$$


and their ratios

$$
r_{j, k}=d_{j, k+1} / d_{j, k}
$$

We will show that $g$ is $C^{1}$ if these ratios converge to 1 geometrically as $j \rightarrow \infty$. In this, we are building on the idea of [15], but, unlike in [15], we show this without assuming anything about the form of the scheme being used, only that it preserves monotonicity.

When working with a ratio $r>0$ it will be convenient to use the notation

$$
r^{*}:=\max \{r, 1 / r\} \geq 1 .
$$

It is useful to note that $|r-1| \leq r^{*}-1$. Let

$$
R_{j}:=\max _{k} r_{j, k}^{*}
$$

\section{Theorem 1 If}

$$
R_{j+1} \leq R_{j}, \quad j \in \mathbb{N}_{0},
$$

and if there is some positive constant $\lambda<1$ such that

$$
R_{j+2}-1 \leq \lambda\left(R_{j}-1\right), \quad j \in \mathbb{N}_{0},
$$

then the limit $g$ in (2) exists and is $C^{1}$.

If we define

$$
e_{j}:=R_{j}-1,
$$

then the first two inequalities in $R_{j}$ imply that

$$
e_{j+1} \leq e_{j}, \quad e_{j+2} \leq \lambda e_{j},
$$

and so

$$
e_{2 j+1} \leq e_{2 j} \leq \lambda^{j} e_{0},
$$

which shows that $e_{j} \rightarrow 0$ geometrically.

Proof. A standard condition for $C^{1}$ continuity $[3,9,19,20]$ is that there are constants $C$ and $\mu<1 / 2$ such that

$$
\max _{k}\left|g_{j, k-1}-2 g_{j, k}+g_{j, k+1}\right| \leq C \mu^{j}
$$

Since

$$
g_{j, k-1}-2 g_{j, k}+g_{j, k+1}=d_{j, k-1}\left(r_{j, k-1}-1\right),
$$


it follows from (4) that (6) holds with $\mu=\sqrt{\lambda} / 2$ if there is a constant $C^{\prime}>0$ such that

$$
d_{j}:=\max _{k} d_{j, k} \leq C^{\prime} 2^{-j} .
$$

To show this, observe that

$$
\begin{aligned}
d_{j+1,2 k} & =d_{j, k} /\left(1+r_{j+1,2 k}\right), \\
d_{j+1,2 k+1} & =d_{j, k} /\left(1+1 / r_{j+1,2 k}\right),
\end{aligned}
$$

and therefore,

$$
d_{j+1} \leq \frac{1}{1+1 / R_{j+1}} d_{j}=\frac{1+e_{j+1}}{2+e_{j+1}} d_{j} \leq \frac{1}{2}\left(1+\frac{e_{j+1}}{2}\right) d_{j} \leq \frac{1}{2} \exp \left(\frac{e_{j+1}}{2}\right) d_{j} .
$$

Hence, applying this recursively, we see that (7) holds with

$$
C^{\prime}=\exp \left(\frac{e_{1}+e_{2}+\cdots}{2}\right) d_{0}
$$

and $C^{\prime}<\infty$ due to $(5)$.

\section{The scheme of Kuijt and van Damme}

Consider now the following non-linear, four-point scheme, studied by Kuijt and van Damme [15]:

$$
\begin{aligned}
g_{j+1,2 k} & =g_{j, k}, \\
g_{j+1,2 k+1} & =\frac{g_{j, k}+g_{j, k+1}}{2}+\frac{d_{j, k}}{4} Q\left(r_{j, k-1}, r_{j, k}\right),
\end{aligned}
$$

where

$$
Q(x, y)=\frac{1}{1+x}-\frac{1}{1+1 / y}, \quad x, y>0 .
$$

Since $|Q(x, y)|<1$ the scheme clearly preserves monotonicity.

Theorem 2 Kuijt and van Damme's scheme (8-9) is $C^{1}$. 
Proof. The scheme for the first order differences of (8-9) is

$$
\begin{aligned}
d_{j+1,2 k} & =\frac{1}{2} d_{j, k}+\frac{d_{j, k}}{4} Q\left(r_{j, k-1}, r_{j, k}\right), \\
d_{j+1,2 k+1} & =\frac{1}{2} d_{j, k}-\frac{d_{j, k}}{4} Q\left(r_{j, k-1}, r_{j, k}\right),
\end{aligned}
$$

and so there is a scheme for the ratios:

$$
\begin{aligned}
r_{j+1,2 k} & =F\left(r_{j, k-1}, r_{j, k}\right), \\
r_{j+1,2 k+1} & =G\left(r_{j, k-1}, r_{j, k}, r_{j, k+1}\right),
\end{aligned}
$$

where

$$
\begin{gathered}
F(x, y)=\frac{2-Q(x, y)}{2+Q(x, y)}, \\
G(x, y, z)=y\left(\frac{1+1 / F(x, y)}{1+F(y, z)}\right),
\end{gathered}
$$

and we will apply Theorem 1 . To prove (3), we see from (10-11) that it is sufficient to show that for any $x, y, z>0$, if $x^{*}, y^{*}, z^{*} \leq R$ for some $R \geq 1$ then

$$
F(x, y)^{*} \leq R, \quad \text { and } \quad G(x, y, z)^{*} \leq R .
$$

To this end, observe that $F$ has the 'reciprocal symmetry' property,

$$
1 / F(x, y)=F(1 / y, 1 / x),
$$

which we can see from the fact that

$$
-Q(x, y)=Q(1 / y, 1 / x)
$$

and so $G$ also has 'reciprocal symmetry':

$$
1 / G(x, y, z)=G(1 / z, 1 / y, 1 / x) .
$$

Therefore (14) holds if

$$
F(x, y) \leq R \quad \text { and } \quad G(x, y, z) \leq R .
$$

Considering the first of these inequalities, observe that $Q(x, y)$ is decreasing in both $x$ and $y$, and therefore $F(x, y)$ is increasing in both $x$ and $y$, and so

$$
F(x, y) \leq F(R, R)=: C_{R},
$$


and since

$$
Q(R, R)=\frac{1-R}{R+1}
$$

we find

$$
C_{R}=\frac{3 R+1}{R+3} .
$$

Since $C_{R} \leq R$, this proves the bound on $F$. Considering the second inequality in (17), since $G$ is decreasing in $x$ and $z$,

$$
G(x, y, z) \leq G(1 / R, y, 1 / R)
$$

From (12),

$$
F(x, y)=\frac{1+2 x+2 y+3 x y}{3+2 x+2 y+x y}
$$

and it follows that $F$ has the symmetry,

$$
F(x, y)=F(y, x)
$$

and so

$$
G(x, y, x)=y / F(x, y) .
$$

The right hand side is increasing in $y$ for all $x, y>0$ because it can be expressed as the product of the two terms

$$
\frac{y}{1+2 x+2 y+3 x y}, \quad 3+2 x+2 y+x y,
$$

both of which are increasing in $y$. Therefore,

$$
G(1 / R, y, 1 / R)=y / F(1 / R, y) \leq R / F(1 / R, R)=R,
$$

from which the second inequality in (17) follows.

Having now established (3), we turn to (4). Taking two steps of the ratio scheme gives

$$
\begin{aligned}
r_{j+2,4 k} & =G_{0}\left(r_{j, k-2}, r_{j, k-1}, r_{j, k}\right), \\
r_{j+2,4 k+1} & =G_{1}\left(r_{j, k-2}, r_{j, k-1}, r_{j, k}, r_{j, k+1}\right), \\
r_{j+2,4 k+2} & =G_{2}\left(r_{j, k-1}, r_{j, k}, r_{j, k+1}\right), \\
r_{j+2,4 k+3} & =G_{3}\left(r_{j, k-1}, r_{j, k}, r_{j, k+1}\right),
\end{aligned}
$$


where

$$
\begin{aligned}
G_{0}(a, b, c) & =F(G(a, b, c), F(b, c)), \\
G_{1}(a, b, c, d) & =G(G(a, b, c), F(b, c), G(b, c, d)), \\
G_{2}(b, c, d) & =F(F(b, c), G(b, c, d)), \\
G_{3}(b, c, d) & =G(F(b, c), G(b, c, d), F(c, d)) .
\end{aligned}
$$

Since both $F$ and $G$ have reciprocal symmetry, i.e. satisfy (15) and (16), it follows that the functions $G_{i}, i=0,1,2,3$, also have reciprocal symmetry and so a sufficient condition for (4) to hold is that there is some $\lambda<1$ such that if $a^{*}, b^{*}, c^{*}, d^{*} \leq R$ then

$$
G_{i}-1 \leq \lambda(R-1), \quad i=0,1,2,3 .
$$

Consider first $G_{0}$ and $G_{2}$. Since

$$
F(R, R)-1=C_{R}-1=\frac{2(R-1)}{R+3} \leq \frac{1}{2}(R-1),
$$

it follows that $(22)$ holds for $i=0,2$ with $\lambda=1 / 2$. Consider next $G_{1}$. Using (18) and (21), we find

$$
G_{1}(a, b, c, d) \leq G(1 / R, y, 1 / R)=y / F(1 / R, y),
$$

where $y=F(b, c) \leq C_{R}$, and so

$$
G_{1}(a, b, c, d) \leq C_{R} / F\left(1 / R, C_{R}\right) .
$$

Since

$$
Q\left(1 / R, C_{R}\right)=\frac{R-1}{4(1+R)}
$$

we find

$$
1 / F\left(1 / R, C_{R}\right)=D_{R}:=\frac{9 R+7}{7 R+9}
$$

Since

$$
C_{R} D_{R}-1=E_{R}(R-1)
$$

where

$$
E_{R}=\frac{20(R+1)}{(R+3)(7 R+9)}
$$


and

$$
E_{R}=\frac{5}{8}\left(1-\frac{(7 R+5)(R-1)}{(R+3)(7 R+9)}\right) \leq \frac{5}{8},
$$

this establishes (22) for $i=1$ with $\lambda=5 / 8$.

Finally consider $G_{3}$. Using (18) and (21), we find

$$
G_{3}(b, c, d) \leq G\left(1 / C_{R}, y, 1 / C_{R}\right)=y / F\left(1 / C_{R}, y\right),
$$

where $y=G(b, c, d) \leq R$, and so

$$
G_{3}(b, c, d) \leq R / F\left(1 / C_{R}, R\right)=R / D_{R}
$$

Since

$$
R / D_{R}-1=F_{R}(R-1)
$$

where

$$
F_{R}=\frac{7(R+1)}{9 R+7}=\frac{7}{8}\left(1-\frac{R-1}{9 R+7}\right) \leq \frac{7}{8},
$$

this establishes (22) for $i=3$ with $\lambda=7 / 8$, and hence (4) with $\lambda=7 / 8$.

\section{The scheme of Sabin and Dodgson}

In their four-point circle-preserving curve scheme, Sabin and Dodgson [17] choose where to locate new points in terms of old ones in a way which can be viewed as choosing parameter values. For functional data the parametervalue scheme defines the new value $g_{j+1,2 k+1}$ by the equation

$$
\frac{d_{j+1,2 k+1}}{d_{j+1,2 k}}=\sqrt{\frac{d_{j, k}+d_{j, k+1}}{d_{j, k-1}+d_{j, k}}} .
$$

This scheme trivially preserves monotonicity, and it also admits a ratio scheme, which is as for Kuijt and van Damme's scheme, but with $F$ in (10) replaced by

$$
F(x, y)=\sqrt{\frac{1+y}{1+1 / x}} .
$$

Theorem 3 Sabin and Dodgson's scheme (23) is $C^{1}$. 
Proof. The function $F$ in (24) has reciprocal symmetry (15), and thus to prove (3), it is sufficient to establish the two inequalities in (17). Considering first $F$, we find

$$
F(x, y) \leq F(R, R)=\sqrt{R} \leq R .
$$

Considering $G$, inequality (19) holds as for Kuijt and van Damme's scheme, but $F$ in (24) does not have the symmetry (20) and so the equation for $G(x, y, x)$ in $(21)$ does not hold. Nevertheless we will show that $G(x, y, x)$ is increasing in $y$ for all $x, y>0$. To do this we can use the property of $F$ in (24) that

$$
F(x, y) F(y, x)=\sqrt{x y}
$$

to eliminate the term $F(y, x)$ from the expression for $G(x, y, x)$ in (13), giving

$$
\frac{1}{G(x, y, x)}=\frac{1}{1+F(x, y)}\left(\frac{F(x, y)}{y}+\sqrt{\frac{x}{y}}\right)
$$

which is decreasing in $y$ since $F(x, y) / y$ is clearly decreasing in $y$. Therefore, since $F(1 / R, R)=1$,

$$
G(x, y, z) \leq G(1 / R, R, 1 / R)=R .
$$

To demonstrate (4) we will again establish the four inequalities in (22). Since

$$
F(R, R)-1=\sqrt{R}-1 \leq \frac{1}{2}(R-1),
$$

inequality (22) holds for $i=0,2$ with $\lambda=1 / 2$. Considering $G_{1}$ and $G_{3}$,

$$
\begin{aligned}
G_{1}(a, b, c, d) & \leq G\left(1 / R, C_{R}, 1 / R\right)=D_{R}, \\
G_{3}(b, c, d) & \leq G\left(1 / C_{R}, R, 1 / C_{R}\right)=E_{R},
\end{aligned}
$$

where $D_{R}$ and $E_{R}$ can be expressed as

$$
D_{R}=\frac{\alpha R^{3 / 4}+\beta R^{1 / 2}}{\alpha+\beta}, \quad E_{R}=\frac{\alpha R+\beta R^{3 / 4}}{\alpha+\beta},
$$

with

$$
\alpha=\sqrt{R+1}, \quad \beta=\sqrt{R+R^{1 / 2}} .
$$

Since these are affine combinations with $R^{3 / 4} \geq R^{1 / 2}, R \geq R^{3 / 4}$, and $\beta \geq \alpha$, we deduce that

$$
D_{R} \leq \frac{R^{3 / 4}+R^{1 / 2}}{2}, \quad E_{R} \leq \frac{R+R^{3 / 4}}{2}
$$



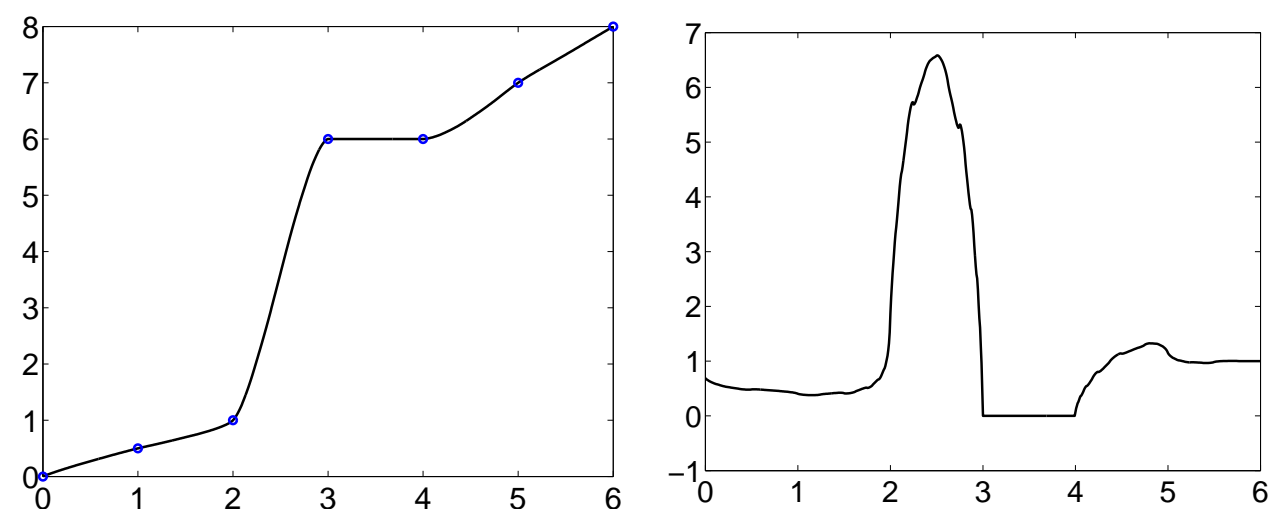

Figure 1: Limit and derivative of Kuijt and van Damme's scheme

Using the fact that for any $\lambda \in[0,1]$,

$$
R^{\lambda}-1 \leq \lambda(R-1),
$$

it follows that (22) holds for $i=1,3$ with $\lambda=5 / 8,7 / 8$ respectively, and hence (4) holds with the same constant $\lambda=7 / 8$ as in Kuijt and van Damme's scheme.

\section{Numerical examples and final remarks}

Figure 1 shows the limit function of Kuijt and van Damme's scheme applied to the data set used in their paper [15]. Figure 2 shows the limit function and derivative of Sabin and Dodgson's scheme applied to the same data. For comparison, Figure 3 shows the limit function and derivative of the linear four-point scheme of [6] and [8], which gives a limit function that is clearly not monotonic.

\section{$5.1 \quad$ Non-strict monotonicity}

The schemes in Sections 3 and 4 extend in a natural way to data that is not strictly increasing. Both rules for the new value $g_{j+1,2 k+1}$ are valid unless all four values $g_{j, k-1}, \ldots, g_{j, k+2}$ are equal, in which case we can define $g_{j+1,2 k+1}$ to be this same value. 

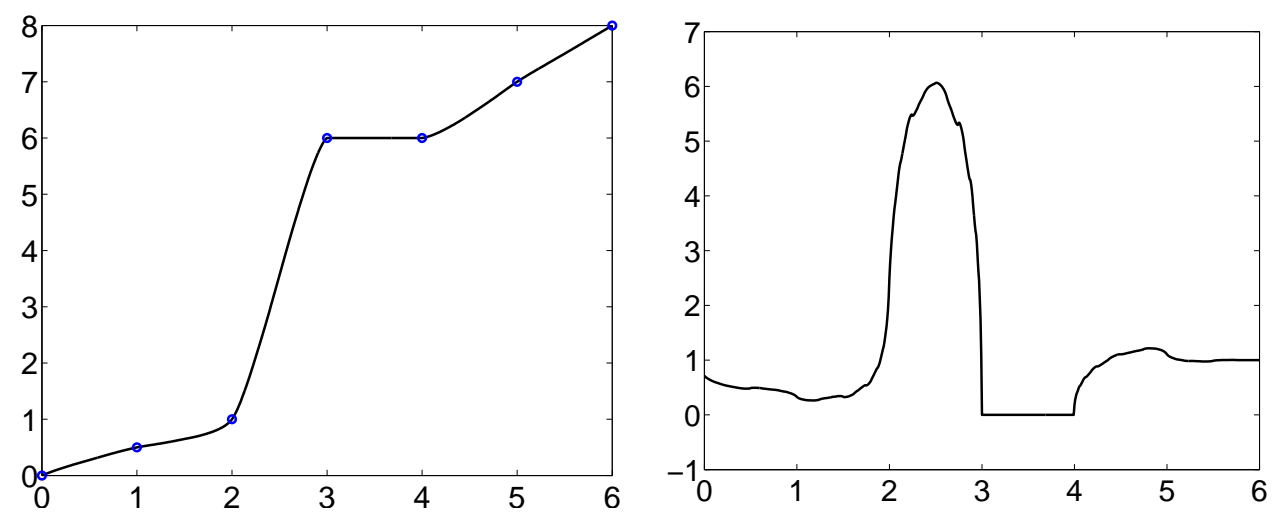

Figure 2: Limit and derivative of Sabin and Dodgson's scheme
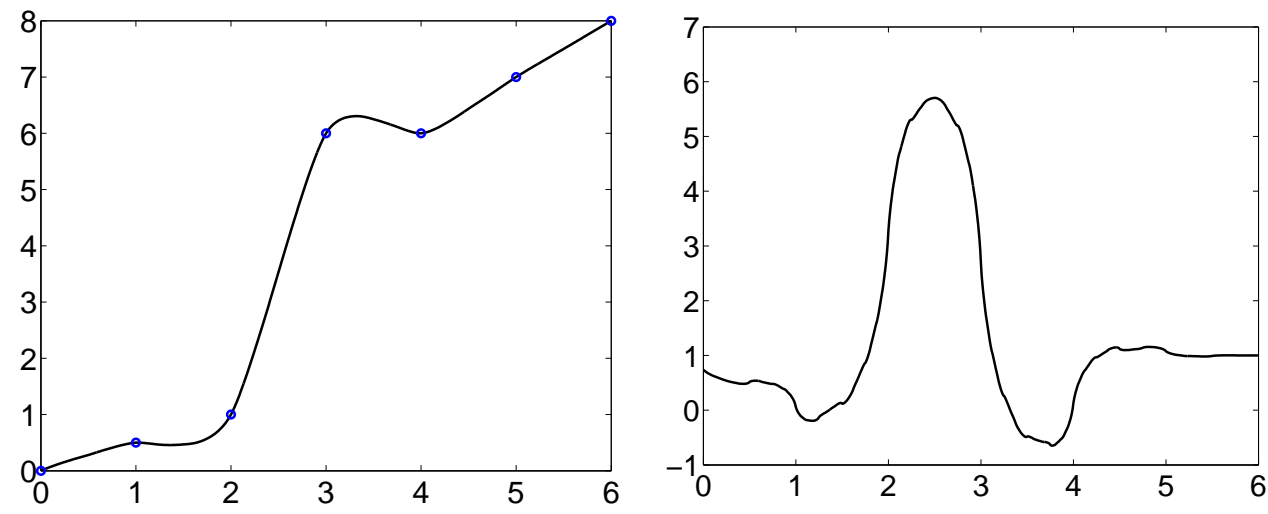

Figure 3: Limit and derivative of the linear four-point scheme

\subsection{Hölder regularity}

Since we have shown that (4) holds for both schemes with $\lambda=7 / 8$ it follows that the Hölder regularity of both schemes is at least $C^{1+\alpha}$, where

$$
\alpha=-\log _{2}(\sqrt{7 / 8}) \approx 0.0963 .
$$

Our numerical tests suggest that both schemes have Hölder regularity $C^{2-\epsilon}$ for any small $\epsilon>0$. This is interesting in comparison with the regularity of the convexity-preserving scheme of [14] and [10] which, according to Yu [20] varies between $C^{1}$ and $C^{2-\epsilon}$ depending on the data. 


\subsection{Application to subdivision on irregular data}

The schemes in this paper can be applied to the subdivision of data $\left(f_{k}, h_{k}\right)$ where function values $h_{k}, k=0,1,2, \ldots, n$, are associated with parameter values $f_{k}$. In this context, the values $g_{j+1,2 k+1}$ are the new parameter values of points which are introduced on level $j$.

The linear four-point scheme $[6,8]$ has been shown to have Hölder regularity $C^{2-\epsilon}$ for the uniform case $f_{k}=k$ and the semi-regular parameterization $g_{j+1,2 k+1}=\left(g_{j, k}+g_{j, k+1}\right) / 2[18]$. The same is true for an arbitrary irregular parametrization (1), as long as the newly inserted values satisfy a condition that ensures they are not too far from the interval midpoint [4]. By proving (5), in this paper we show that the considered schemes are asymptotically uniform: they insert values which are arbitrarily close to interval midpoints as $j \rightarrow \infty$. We can therefore use these results to show that the linear four-point scheme, using either Kuijt and van Damme's scheme or Sabin and Dodgson's scheme to choose parameter values, has the same Hölder regularity $C^{2-\epsilon}$ for irregular data as in the uniform case.

\subsection{A fourth root scheme}

While working on Theorems 2 and 3 we discovered another interpolatory scheme that preserves monotonicity and is $C^{1}$. The scheme chooses the new value $g_{j+1,2 k+1}$ by the equation

$$
\frac{d_{j+1,2 k+1}}{d_{j+1,2 k}}=\left(\frac{d_{j, k+1}}{d_{j, k-1}}\right)^{1 / 4},
$$

and so $F$ in (12) is replaced by

$$
F(x, y)=(x y)^{1 / 4}
$$

The proof that this scheme is $C^{1}$ is similar to that of Sabin and Dodgson's scheme, but simpler, and the limit function also looks similar. However, the scheme becomes unstable when strictly monotonic data approaches nonstrictly monotonic data: as strictly monotonic data approaches the four limiting values $g_{j, k-1}=g_{j, k}=0$ and $g_{j, k+1}=g_{j, k+2}=1$ the limiting value $g_{j+1,2 k+1}$ can be anywhere in the range $[0,1]$. This could be viewed as a drawback in practical applications. 


\section{References}

[1] U. H. Augsdörfer, N. A. Dodgson, and M. A. Sabin, Variations on the four-point subdivision scheme, Comp. Aided Geom. Design 27 (2010), 78-95.

[2] C. Beccari, G. Casciola, L. Romani, Polynomial-based non-uniform interpolatory subdivision with features control, J. Comput. Appl. Math. 235 (2011), 4754-4769.

[3] A. S. Cavaretta, W. Dahmen, and C. A. Micchelli, Stationary subdivision, in Memoirs of the American Mathematical Society (1991), 186 pp.

[4] I. Daubechies, I. Guskov, and W. Sweldens, Regularity of irregular subdivision, Constr. Approx. 15 (1999), 381-426.

[5] R. Delbourgo and J. A. Gregory, $C^{2}$ rational quadratic spline interpolation to monotonic data, IMA J. Numer. Anal. 3 (1983), 141-152.

[6] S. Dubuc, Interpolation through an iterative scheme, J. Math. Anal. Appl. 114 (1986), 185-204.

[7] N. Dyn, M. Floater, and K. Hormann, Four-point curve subdivision based on iterated chordal and centripetal parameterizations, Comput. Aided Geom. Design 26 (2009), 279-286.

[8] N. Dyn, J. Gregory, and D. Levin, A four-point interpolatory subdivision scheme for curve design, Comput. Aided Geom. Design 4 (1987), $257-$ 268.

[9] N. Dyn and D. Levin, Subdivision schemes in geometric modelling, Acta Numerica 11 (2002), 73-144.

[10] M. S. Floater and C. A. Micchelli, Nonlinear stationary subdivision, in Approximation Theory: in Memory of A. K. Varma, N. K. Govil, R. N. Mohapatra, Z. Nashed, A. Sharma and J. Szabados (eds.), Marcel Dekker, New York (1998), 209-224.

[11] F. N. Fritsch and J. Butland, A method for constructing local monotone piecewise cubic interpolants, SIAM J. Sci. Stat. Comp. 5 (1984), 200204. 
[12] F. N. Fritsch and R. E. Carlson, Monotone piecewise cubic interpolation, SIAM J. Numer. Anal. 17 (1980), 238-246.

[13] J. A. Gregory and R. Delbourgo, Piecewise rational quadratic interpolation to monotonic data, IMA J. Numer. Anal. 2 (1982), 123-130.

[14] F. Kuijt and R. van Damme, Convexity preserving interpolatory subdivision schemes, Constr. Approx., 14 (1998), 609-630.

[15] F. Kuijt and R. van Damme, Monotonicity preserving interpolatory subdivision schemes, J. Comput. Appl. Math. 101 (1999), 203-229.

[16] F. Kuijt and R. van Damme, Shape-preserving interpolatory subdivision schemes for nonuniform data, J. Approx. Theory 114 (2002), 1-32.

[17] M. A. Sabin, N. Dodgson, A circle-preserving variant of the four-point subdivision scheme, in: Dæhlen, M., Morken, K., Schumaker, L.L. (eds.), Mathematical Methods for Curves and Surfaces: Troms $\varnothing 2004$, Nashboro Press, pp. 275-286 (2005).

[18] J. Warren, Binary subdivision schemes for functions over irregular knot sequences, in: Dæhlen, M., Lyche, T., Schumaker, L.L. (eds.), Mathematical Methods in Computer Aided Geometric Design III, Vanderbilt Univ. Press, pp. 543-562 (1995).

[19] G. Xie and T. P.-Y. Yu, Smoothness analysis of nonlinear subdivision schemes of homogeneous and affine invariant type, Const. Approx., 22 (2005), 219-254.

[20] T. P.-Y. Yu, How data dependent is a nonlinear subdivision scheme? A case study based on convexity preserving subdivision, SIAM J. Numer. Anal. 44 (2006), 936-948. 